Conclusion: Our findings show substantial decline in risk of dementia in patients with RA onset in the 2000 s as compared to 1980 s, including when compared to the general population comparators. This decline coincides with the advent of novel biologic treatments for RA. Further studies should investigate this association using manual verification rather than billing codes for dementia, and should also elucidate the role of inflammation, autoimmunity, and anti-rheumatic treatments in risk of dementia.

Acknowledgements: This work was funded by grants from the National Institutes of Health, NIAMS (R01 AR46849) and NIA (R01 AG068192, R01 AG034676). Disclosure of Interests: Vanessa Kronzer: None declared, Cynthia S. Crowson: None declared, John M Davis III Grant/research support from: Pfizer, Maria Vassilaki Shareholder of: Abbott Laboratories, Johnson and Johnson, Medronic and Amgen, Grant/research support from: Roche, Biogen, Michelle Mielke Consultant of: Biogen and Brain Protection Company, Elena Myasoedova: None declared

DOI: 10.1136/annrheumdis-2021-eular.150

\section{OP0217 IS METACARPOPHALANGEAL-JOINT PAIN AS AN EARLY SYMPTOM OF PATIENTS AT RISK FOR PROGRESSION TO INFLAMMATORY ARTHRITIS EXPLAINED BY MRI-DETECTED SUBCLINICAL INFLAMMATION? - A LARGE OBSERVATIONAL STUDY}

Q. Dumoulin ${ }^{1}$, X. Matthijssen ${ }^{1}$, F. Wouters ${ }^{1}$, A. Van der Helm - van Mil $^{1,2}$, E. Niemantsverdriet. ${ }^{1}{ }^{1}$ Leiden University Medical Center (LUMC), Rheumatology, Leiden, Netherlands; ${ }^{2}$ Erasmus MC, Rheumatology, Rotterdam, Netherlands

Background: Pain in metacarpophalangeal (MCP)-joints in patients presenting with clinically suspect arthralgia (CSA) is one of the clinical features by which patients are considered at risk for progression to inflammatory arthritis (IA). As such this symptom is characteristic for CSA and therefore part of a list of clinical parameters determined by an EULAR-taskforce to identify a more homogeneous group of patients within CSA (the EULAR definition of arthralgia suspicious for progression to RA). MRI-detected subclinical inflammation is known to be present in patients with CSA. In general, arthralgia in CSA can be explained by this subclinical inflammation, however to date, the association of subclinical inflammation with pain in MCP-joints specifically is not clear. Subsequently, it is unknown whether this association differs pertinently when investigated with self-reported pain, or with pain in the form of tenderness at physical examination. Objectives: This study will investigate whether MCP-pain and MCP-joint tenderness are associated with MRI-detected subclinical inflammation in patients with CSA, and more specifically those who have progressed to IA

Methods: Between April 2012- February 2019, 602 patients were consecutively included in the Leiden clinically suspect arthralgia (CSA)-cohort. Follow-up ended when patients developed clinically apparent IA (determined at physical examination), or else after 2-years (median follow-up time 25 months). MCP-joints were assessed for self-reported joint pain by the patient using a mannequin and subsequently for joint tenderness by physical examination. Baseline unilateral MRIs of the MCP (2-5)-joints were scored by two readers, blinded for clinical data, on subclinical inflammation (synovitis, tenosynovitis, osteitis). Associations between MCP-pain or MCP-joint tenderness and MRI-detected subclinical inflammation were studied at patient level by logistic regression analyses, entering the mentioned MRI-detected features separately (univariable) and together (multivariable). Results: $33 \%$ of 227 patients with self-reported MCP-pain had MRI-detected subclinical inflammation and $38 \%$ of 226 patients with MCP-joint tenderness had MRI-detected subclinical inflammation. Self-reported MCP-joint pain was univariable associated with subclinical inflammation and synovitis in particular (OR 2.00, 95\% Cl: 1.21-3.30, OR 2.87, 95\% Cl: 1.29-6.39). In multivariable analysis this MCP-pain was associated with synovitis (OR 2.54, 95\% Cl: 1.12-5.77). MCP-joint tenderness was univariable associated with subclinical inflammation, and synovitis and tenosynovitis in particular (OR 1.84, 95\% Cl: $1.29-2.63$, OR $1.76,95 \% \mathrm{Cl}$ : $1.10-2.81$, OR $1.69,95 \% \mathrm{Cl}: 1.12-2.55$, respectively). In multivariable analysis, tenosynovitis remained significant (OR 1.54, 95\% Cl: 1.00-2.36). Of all patients with self-reported MCP-joint pain who developed IA, 50\% had MRI-detected subclinical inflammation. For MCP-joint tenderness this was $61 \%$. Patients with MCP-joint tenderness without subclinical inflammation who developed IA, developed clinical arthritis at a joint that was not scanned (85\%), hence they may have had subclinical inflammation that was not imaged. The other $15 \%$ did develop arthritis in an MCP-joint, suggesting that subclinical inflammation developed after CSA-onset. Conclusion: Arthralgia in the MCP-joints is associated with subclinical inflammation in CSA, in particular with synovitis and tenosynovitis. The prevalence of subclinical inflammation is highest for tender joints at physical examination; this can be acknowledged when applying the EULAR definition of arthralgia suspicious for progression to RA.

Disclosure of Interests: None declared

DOI: 10.1136/annrheumdis-2021-eular.1597

\section{Unrevealing the impact of PsA and comorbidity prevention}

\section{OP0218 \\ MORTALITY IN PATIENTS WITH PSORIATIC ARTHRITIS} IN SWEDEN

S. Exarchou ${ }^{1}$, D. DI Giuseppe ${ }^{2}$, G. M. Alenius ${ }^{3}$, E. Klingberg ${ }^{4,5}$, V. Sigurdardottir ${ }^{6,7}$, S. Wedrén ${ }^{2,8}$, U. Lindström ${ }^{5}$, C. Turesson ${ }^{1}$, L. T. H. Jacobsson ${ }^{5}$, J. Askling ${ }^{2}$, J. K. Wallman ${ }^{9,10} .{ }^{1}$ Lund University, Department of Clinical Sciences Malmö, Rheumatology, Lund, Sweden; ${ }^{2}$ Karolinska Institutet, Department of Medicine Solna, Clinical Epidemiology Division, Stockholm, Sweden; ${ }^{3}$ Umeå University, Department of Public Health and Clinical Medicine/Rheumatology, Umeå, Sweden; ${ }^{4}$ Sahlgrenska University Hospital, Department of Rheumatology, Gothenburg, Sweden; ${ }^{5}$ Sahlgrenska Academy, University of Gothenburg, Department of Rheumatology and Inflammation Research, Gothenburg, Sweden; ${ }^{6}$ Falun Hospital, Department of Rheumatology, Falun, Sweden; ${ }^{7}$ Uppsala University, Centre for Clinical Research Dalarna, Uppsala, Sweden; ${ }^{8}$ Karolinska University Hospital, Department of Rheumatology, Stockholm, Sweden; ${ }^{9}$ Skåne University Hospital, Department of Rheumatology, Lund, Sweden; ${ }^{10}$ Lund University, Department of Clinical Sciences Lund, Rheumatology, Lund, Sweden

Background: In contrast to the increased mortality reported in other inflammatory diseases such as rheumatoid arthritis and psoriasis, prior mortality studies in psoriatic arthritis ( $\mathrm{PsA}$ ) have shown inconsistent results.

Objectives: To compare all-cause mortality between PsA patients in Sweden and matched general population controls, and to describe cause of death distributions in the two groups.

Methods: All individuals in Sweden with $\geq 1$ main diagnosis of PsA (ICD-10: L40.5 M07.0-M07.3) from outpatient visits to rheumatology or internal medicine clinics a age $\geq 18$ years $(y)$ 2001-2017 were identified from the Swedish National Patient Register. Each case was matched to 5 general population controls based on sex, county and age in the year of the first registered arthritis diagnosis for the case. Cases and controls were followed from 1 Jan, 2007, or from first PsA diagnosis thereafter for index cases, until first occurrence of death (data from the Swedish Cause of Death Register), emigration or $31 \mathrm{Dec}, 2018$. Mortality was assessed overall, as well as stratified by sex (45\% males) and disease duration (PsA diagnosis prior to 2007 [38\% of cases] vs. 2007-2017), using matched Cox proportional hazard regression, or - in case the Cox assumption regarding proportionality did not hold - matched Breslow test. To account for potential PsA misclassification (in a previous validation study, $86 \%$ of 400 cases fulfilled PsA classification criteria), a sensitivity analysis was performed by randomly replacing $20 \%$ of cases with one of their own controls. Moreover, incidence rate ratios (IRR) of death were calculated overall and stratified by sex, disease duration and age. Finally, causes of death (from the Cause of Death Register) were described for PsA cases and controls.

Results: Over the 12y follow-up, 3121 deaths occurred among 33036 PsA cases (268 402 person-years at risk) and 12884 deaths among 161144 controls (1 302 250 person-years), resulting in an increased mortality among the PsA cases (HR $1.11[95 \% \mathrm{Cl} 1.07-1.16], \mathrm{p}<0.001$, Figure and Table; sensitivity analysis HR 1.09 [1.05-1.14]). The increased mortality was seen mainly among female PsA cases and among cases with longer disease duration (Figure; Table). IRR:s of death were significantly increased for all ages except $<40 \mathrm{y}$, with the numerically highest point-estimates for ages 40-49y and 50-59y (Table). Cause of death frequencies among the PsA cases/controls: cardiovascular disease $29 / 27 \%$; diabetes mellitus $2.1 / 2.5 \%$; chronic kidney disease $0.4 / 0.3 \%$; infection $5.7 / 4.5 \%$; chronic pulmonary disease $5.1 / 4.1 \%$; malignancy $29 / 34 \%$; suicide $2.3 / 2.0 \%$; other $27 / 26 \%$.

Conclusion: In this nationwide $12 \mathrm{y}$ assessment, the mortality risk among PsA patients in Sweden was increased by around $10 \%$ as compared to the general population, mainly driven by increased risks among females and patients with longer disease duration. Cause of death distributions were numerically similar between PsA cases and controls.

\section{References:}
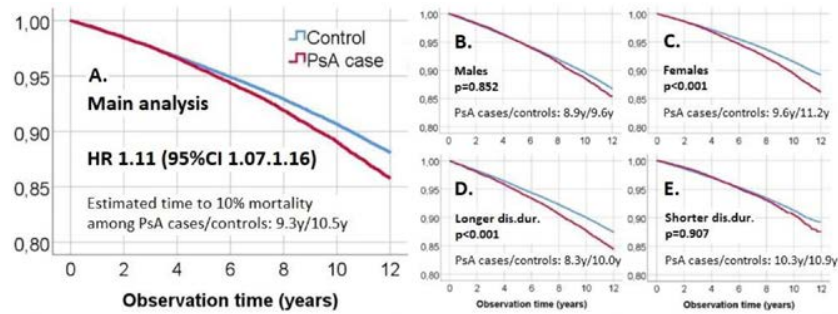

Figure. Kaplan-Meier survival curves displaying all-cause mortality among PsA cases and matched general population controls in Sweden overall (A) and in subgroups (B-E). Estimated time to $10 \%$ mortality are displayed in the respective graphs. A. Main analysis. B. Males, analysed by Breslow test. C. Females, HR disp (95\% 1 16-1 30) D. Lo duration, analysed by Breslow test. Dis.dur, disease duration; PsA, psoriatic arthritis; $y$, years. 